\title{
POTENCIAL ANTINOCIEPTIVO DO ÓLEO ESSENCIAL DE Lippia bromelyana Moldenke (Verbenaceae) EM CAMUNDONGOS.
}

\section{Alanna Caroline de Jesus Dias ${ }^{1}$; Marilene Lopes da Rocha ${ }^{2}$; Débora Maria Marchesine}

de Almeida ${ }^{3}$ e Jonathan Lima Rios Teles ${ }^{4}$

1. Bolsista PIBIC/CNPq, Graduando em Ciências Biológicas, Universidade Estadual de Feira de Santana, e-mail: nany.acjd@gmail.com.br

2. Orientador, Departamento de Ciências Biológicas, Universidade Estadual de Feira de Santana, e-mail: mlrochaph@gmail.com.br

3. Participante do Programa de Pós-graduação em Biotecnologia , Departamento de Ciências Biológicas, Universidade Estadual de Feira de Santana, e-mail: debora.mma01@ gmail.com.br 4.Bolsista PIBIC/CNPq, Graduando em Ciências Biológicas, Departamento de Ciências Biológicas, Universidade Estadual de Feira de Santana, e-mail: jonhlrios@ gmail.com.br

PALAVRAS-CHAVE: Antinociceptivo; óleo essencial; Lippia spp

\section{INTRODUÇÃO}

No Brasil, o uso de plantas medicinais é bastante difundido e, em grande parte foi influenciado pela tradição popular das culturas indígenas nativas, africana, asiática e européia (MACIEL et al., 2002; MARTINS et al., 2000; RATES, 2001). A região Nordeste, que abriga o bioma caatinga, possui muitas espécies de plantas nativas que são empregadas na medicina popular para o tratamento de diversas doenças (ALBUQUERQUE et al., 2005). Dentre as quais, as do gênero Lippia (Verbenaceae), empregadas tradicionalmente no tratamento de distúrbios relacionados ao sistema respiratório e a problemas gastrointestinais.

Diversos estudos (GOMES, et al., 2011; GUILHON et al., 2011; GUIMARÃES et al., 2012; HALDAR et al., 2012; MENDES et al., 2010; PEREIRA et al., 2013; RIELLA et al., 2012) têm comprovado certas propriedades medicinais dessas plantas, no entanto, mesmo havendo uma grande diversidade de espécies de Lippia com propriedades farmacológicas, inexistem estudos científicos comprovando tais atributos para Lippia bromelyana Moldenke, em particular.

Diante disso, a realização deste trabalho se justifica na necessidade de investigar as propriedades farmacológicas do óleo essencial desta planta no que se refere à analgesia a fim de fornecer dados científicos que possibilitem sua utilização terapêutica segura e a realização de estudos mais aprofundados.

\section{MATERIAL E MÉTODOS OU METODOLOGIA}

1. COLETA DO MATERIAL BOTÂNICO 
A espécie será coletada no campo experimental do Horto Florestal da UEFS e o tratamento convencional de herborização seguirá o descrito em Mori et al. (1989).

\section{OBTENÇÃO DO ÓLEO ESSENCIAL DE L. bromelyana}

Após trituração do material vegetal, o óleo essencial será extraído por meio da hidrodestilação, em triplicata, utilizando um aparelho tipo Clevenger, com duração de três horas.

\section{ANIMAIS DE EXPERIMENTAÇÃO}

Serão utilizados camundongos Mus musculus (LINNAEUS, 1758), machos, adultos, pesando entre 25 e 30 gramas, obtidos da colônia do Biotério Central da Universidade Estadual de Feira de Santana.O presente trabalho será cadastrado e submetido à aprovação pela Comissão de Ética no Uso de Animais (CEUA) da Universidade estadual de Feira de Santana.

4. TRIAGEM FARMACOLÓGICA COMPORTAMENTAL COM DETERMINAÇÃO DA TOXICIDADE AGUDA (DL $\left.{ }_{50 \%}\right)$

3. TESTE PARA INVESTIGAÇÃO DA ATIVIDADE MOTORA NA BARRA GIRATÓRIA (ROTA-ROD)

Os animais serão colocados numa barra giratória, a fim de verificar o efeito do relaxamento muscular ou da incoordenação motora produzido pelo $\mathrm{OE}$ de $L$. bromelyana (CARLINI; BURGOS, 1979; MATTEI; FRANCA, 2006).

\section{TESTES FARMACOLÓGICOS ESPECÍFICOS PARA AVALIAÇÃO DA ATIVIDADE ANTINOCIEPTIVA}

Os testes de avaliação da atividade antinociceptiva do OE de L. bromelyana seguirão o mesmo padrão de formação, com os camundongos ( $\mathrm{n}=8$ por grupo), distribuídos em controle, padrão (morfina 10mg/kg, i.p.) e experimentais $(75,150$ e 300 mg/kg, i.p.).

5. AVALIÇÃO dO ENVOLVIMENTO DO SISTEMA OPIÓDE NA AÇÃO DO ÓLEO ESSENCIAL DE L. bromelyana

\section{RESULTADOS E/OU DISCUSSÃO}

1. TESTE PARA INVESTIGAÇÃO DA ATIVIDADE MOTORA NA BARRA GIRATÓRIA (ROTA-ROD) 


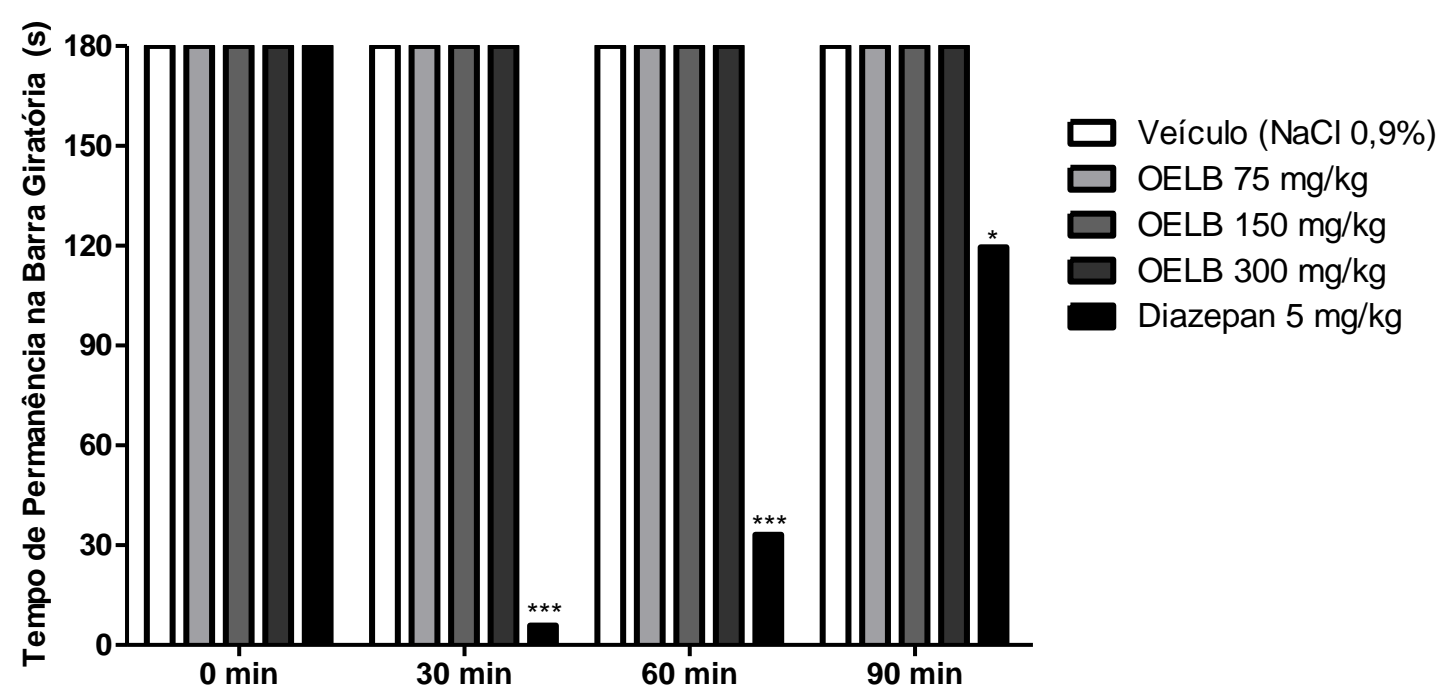

Figura 3. Efeito do OELB (75, 150 ou $300 \mathrm{mg} / \mathrm{kg}$, i.p.) e diazepan (5 mg/kg, i.p.) no teste do rota-rod. Os valores foram expressos em média \pm e.p.m. $(\mathrm{n}=8)$. ${ }^{*} p<0,05$ foram considerados significativos quando comparados com o grupo controle (ANOVA seguido do teste de Tukey).

\section{TESTES FARMACOLÓGICOS ESPECÍfICOS PARA AVALIAÇÃO DA ATIVIDADE ANTINOCIEPTIVA}

\section{AVALIÇÃO DO ENVOLVIMENTO DO SISTEMA OPIÓdE NA AÇÃO DO ÓLEO ESSENCIAL DE L. bromelyana}

Os dados sugerem que OELB mostrou efeito antinociceptivo tanto por ação central quanto periférica em camundongos, inclusive a participação do sistema opioidérgico, uma vez que a naloxona foi capaz de reverter a ação do OE reduzindo o limiar nociceptivo.

Há evidências de que OELB pode apresentar ação anti-inflamatória em camundongos, visto que na $2^{\mathrm{a}}$ fase do teste da formalina houve redução significativa do tempo de lambida da pata. Além disso, no teste da carragenina, OELB diminuiu significativamente o volume do edema de pata em todas as doses testadas em comparação ao grupo controle.

OELB apresenta como componentes majoritários os terpenos limoneno e óxido de piperitona. Os quais provavelmente estão relacionados às propriedades farmacológicas relatadas neste estudo. Além disso o OE não apresentou efeito tóxico até a dose $300 \mathrm{mg} / \mathrm{kg}$ nem provocou morte.

\section{CONSIDERAÇÕES FINAIS}


O composto estudado apresenta grande potencial biotecnológico, entretanto é necessária a investigação minuciosa de suas propriedades para elucidação de mecanismos de ação mais específicos.

\section{REFERÊNCIAS}

ALBUQUERQUE, U.P. et al. Medicinal plants of the caatinga (semi-arid) vegetation of NE Brazil: a quantitative approach. Journal of Ethnopharmacology, v. 114, p.325-354, 2007.

CARLINI, E. A.; BURGOS, V. Screening farmacologico de Ansioliticos: Metodologia Laboratorial e comparação entre o diazepam e o clorobenzapam. Revista da Associação Brasileira de Psiquiatria, v. 1, p. 25-31, 1979.

GOMES, S. V. F. et al., Aspectos químicos e biológicos do gênero Lippia enfatizando Lippia gracilis Schauer. Eclética Química, v. 36, São Paulo, 2011.

GUILHON, C. C. et al. Characterisation of the anti-inflammatory and antinociceptive activities and the mechanism of the action of Lippia gracilis essential oil. Journal of Ethnopharmacology, v. 135, p. 406-413, 2011.

GUIMARÃES, A. G.; et al. Phytochemical characterization and antinociceptive effect of Lippia gracilis Schauer. Journal of Natural Medicines, v. 66, p. 428-434, 2012.

HALDAR, S.; et al. In vivo anti-nociceptive and anti-inflammatory activities of Lippia alba. Asian Pacific Journal of Tropical Disease, p. S667-S670, 2012.

MACIEL, M. A. M., et al. Plantas medicinais: a necessidade de estudos multidisciplinares. Química Nova, v. 25, n. 3, p. 429-438, 2002.

MARTINS, E. R.; et al. Plantas Medicinais. Viçosa: Editora UFV, 2000. 220 p.

MATTEI, R.; FRANCA, C. I. F. Testes gerais para confirmar a ação central. In: ALMEIDA, R. N. Psicofarmacologia: fundamentos práticos. 1. ed. Rio de Janeiro: Guanabara Koogan, 2006. cap. 12, p. 138-142.

MENDES, S. S., et al. Evaluation of the analgesic and anti-inflammatory effects of the essential oil of Lippia gracilis leaves. Journal of Ethnopharmacology, v. 129, p. 391-397, 2010.

PEREIRA, S. L. S. et al. Clinical effect of a gel containing Lippia sidoides on plaque and gingivitis control. European Journal of Dentistry, v. 7, 2013.

RATES, S. M. K. Plants as sources of drugs. Toxicon, v. 39, p. 603-613, 2001.

RIELLA, K. R. et al. Anti-inflammatory and cicatrizing activities of thymol, a monoterpene of the essential oil from Lippia gracilis, in rodents. Journal of Ethnopharmacology 143 656-663, 2012. 